\title{
Acesso ao diagnóstico da tuberculose em serviços de saúde do município de Vitória, ES, Brasil
}

\author{
Access to the diagnosis of tuberculosis in health services \\ in the municipality of Vitoria, state of Espírito Santo, Brazil
}

Rafaela Borges Loureiro ${ }^{1}$

Tereza Cristina Scatena Villa ${ }^{2}$

Antônio Ruffino-Netto ${ }^{3}$

Renata Lyrio Peres ${ }^{1}$

Jose Ueleres Braga ${ }^{4}$

Eliana Zandonade ${ }^{5}$

Ethel Leonor Noia Maciel ${ }^{1}$

\footnotetext{
${ }^{1}$ Núcleo de Doenças Infecciosas, Universidade Federal do Espírito Santo. Av. Marechal Campos 1468, Maruípe. 29.040-091.

Vitória ES Brasil. rafaelabloureiro@gmail.com

${ }^{2}$ Departamento de

Enfermagem em Saúde

Pública, Escola de

Enfermagem de Ribeirão

Preto, Universidade de São

Paulo.

${ }^{3}$ Departamento de Medicina

Social, Faculdade de

Medicina de Ribeirão Preto,

Universidade de São Paulo.

${ }^{4}$ Departamento de

Epidemiologia. Instituto de

Medicina Social,

Universidade do Estado do

Rio de Janeiro.

${ }^{5}$ Programa de Pós-

Graduação em Saúde

Coletiva, Universidade

Federal do Espírito Santo.
}

Abstract This study sought to assess the accessibility to the diagnosis of tuberculosis in health services in Victoria, state of Espirito Santo. It featured a cross-sectional study conducted in 2009 of patients with tuberculosis using the Primary Care Assessment Tool and statistical analysis with the Chi-square test $(p<0,05)$. In relation to initial access to care, it was seen that the health service of first access most sought was Primary Care (37.6\%), with most diagnoses occurring in the Tuberculosis Control Program Reference Units (61.3\%). There was evidence of association between first health service accessed and the factors of time delay in: obtaining consultation at the first health service sought ( $p=0.0182)$; diagnosis made by the first health service sought ( $p=$ $0.0001)$; request for sputum exam ( $p=0,0003)$; request for $X$-ray exams ( $p=0.0159)$; referral for $X$-rays at another institution ( $p=0.0001)$; diagnosis by the same health service ( $p=0.0001)$; exams conducted by the same health service that initially diagnosed tuberculosis $(p=0.0018)$; and proximity to the home ( $p=0.0001)$. Therefore, the identification of important gaps in accessibility to diagnosis of tuberculosis seems to be related to the operational difficulties of organization of health care.

Key words Tuberculosis, Access, Accessibility, Health Services, Diagnosis, Primary health care
Resumo Objetivou-se avaliar a acessibilidade ao diagnóstico da tuberculose nos serviços de saúde em Vitória (ES). Estudo transversal realizado em 2009 com doentes de tuberculose, utilizando o instrumento Primary Care Assessment Tool. Análise estatística com Teste Qui-quadrado $(p<0,05)$. Em relação à porta de entrada, notou-se que o serviço de saúde mais procurado foi Atenção Básica $(37,6 \%)$; a maioria dos diagnósticos ocorreu nas Unidades de Referência do Programa de Controle da Tuberculose (61,3\%). Houve evidência de associação entre primeiro serviço de saúde procurado e fatores tempo de demora na obtenção de consulta neste ( $p=0,0182)$, hipótese diagnóstica feita pelo primeiro serviço de saúde procurado ( $p$ $=0,0001)$, solicitação exame de escarro $(p=$ $0,0003)$, solicitação exame de Raios- $X(p=0,0159)$, encaminhamento para Raios- $X$ em outro serviço ( $p=0,0001)$, diagnóstico pelo mesmo serviço de saúde ( $p=0,0001)$, exames realizados no próprio serviço de saúde que diagnosticou tuberculose ( $p=$ $0,0018)$, proximidade do domicílio ( $p=0,0001)$. Portanto, a identificação de lacunas importantes na acessibilidade ao diagnóstico de tuberculose parece estar relacionada às dificuldades operacionais de organização da atenção à saúde.

Palavras-chave Tuberculose, Acesso, Acessibilidade, Serviços de saúde, Diagnóstico, Atenção Primária à Saúde 


\section{Introdução}

No Brasil, o Programa Nacional de Controle da Tuberculose (PNCT), desde 2004, reconhece a importância de ampliar o combate à tuberculose (TB) a todos os serviços de saúde do SUS e, portanto, visa à integração do controle da TB com a atenção básica, incluindo-o como ação do Programa de Saúde da Família para garantir a efetiva ampliação do acesso ao diagnóstico e tratamento ${ }^{1}$.

O comportamento (que segundo Fisbein e Ajzen $^{2}$, é o somatório de crenças e valores) do indivíduo geralmente é o responsável pelo primeiro contato com os serviços de saúde, e a abordagem e o acolhimento dos profissionais de saúde são responsáveis pelos contatos subsequentes. Os profissionais em grande parte definem o tipo e a intensidade de recursos consumidos para resolver os problemas de saúde dos pacientes ${ }^{3}$.

Nesse sentido, o conceito de acesso é central em muitas discussões que têm o intuito de qualificar a organização do serviço de saúde ${ }^{4}$. Seu conceito é multidimensional e exprime um conjunto de características da oferta que facilitam ou limitam a capacidade das pessoas usarem o serviço de saúde quando necessitam 5 .

$\mathrm{O}$ acesso aos serviços de saúde pode ser entendido como "porta de entrada", como o local de acolhimento do usuário no momento de expressão de sua necessidade e, de certa forma, aos caminhos percorridos por ele no sistema na busca da resolução desta ${ }^{6}$. Nessa perspectiva, $\mathrm{o}$ acesso vai além da conexão pura e simples ao conceito de porta de entrada, configura-se como um "dispositivo" transformador da realidade ${ }^{6}$.

Donabedian ${ }^{7}$ opta pelo termo acessibilidade e o define como um dos aspectos do serviço relativo à capacidade de responder às necessidades de saúde de uma determinada população. Nesse sentido, discute as características dos serviços que facilitam ou dificultam o acesso da população.

Frenk ${ }^{8}$ caracteriza a acessibilidade como a relação funcional entre a resistência, isto é, o conjunto de obstáculos (ecológicos, financeiros, organizacionais) para procurar e obter cuidados, e o poder de utilização, ou seja, a capacidade da população de superar tais obstáculos de forma a melhor utilizar os serviços oferecidos, o que caracteriza sua capacidade de resposta.

Palermo ${ }^{9}$ avança nessa discussão, sugerindo ainda que a acessibilidade seja considerada em seus diferentes aspectos: sociocultural, organizacional, geográfico e econômico.

Assim, percebe-se que na literatura há variação no uso da terminologia e conceito de acesso e acessibilidade. Embora haja divergências, em uma concepção ampla, a acessibilidade pode ser definida como o grau de ajuste entre as características da oferta/disponibilidade dos recursos de saúde e as da população, no processo de busca e obtenção da assistência à saúde, resultando da combinação de diversos fatores de dimensões distintas, que podem ser classificados como de ordem sociocultural, organizacional, geográfica, e econômica ${ }^{10-12}$.

Neste estudo, o acesso na avaliação da estrutura dos serviços será utilizado como sinônimo de acessibilidade para indicar o grau de facilidade ou dificuldade com que as pessoas obtêm cuidados de saúde e o conceito de Unglert ${ }^{13}$ e Palermo ${ }^{9}$ será utilizado como referencial para a análise de barreiras financeiras, organizacionais e/ou estruturais ${ }^{14}$ que dificultam o acesso ao diagnóstico de TB.

A acessibilidade ao diagnóstico da TB vem sendo estudada sob estas duas vertentes: a dos doentes e a dos serviços de saúde, mostrando que ambas apresentam características que levam às falhas na detecção precoce dos casos e, consequentemente, à redução das taxas de cura e aumento da carga da doença em todo o mundo ${ }^{15-20}$.

Assim, o objetivo deste estudo foi analisar a acessibilidade dos doentes de TB ao diagnóstico nos diferentes serviços de saúde no município de Vitória, Espírito Santo.

\section{Material e métodos}

Foi realizado um estudo epidemiológico transversal, de caráter exploratório, com abordagem quantitativa, no Município de Vitória (ES). O Município de Vitória é classificado como um dos prioritários do Espírito Santo para o controle da TB e apresenta uma população de 320.156 habitantes, segundo estimativas do IBGE $2009^{21}$.

Atualmente, o município apresenta cerca de $80 \%$ da população coberta pelo Programa de Saúde da Família (PSF) e Programa de Agentes Comunitários de Saúde (PACS). O município é dividido em 6 regiões de saúde com a rede ambulatorial distribuída da seguinte forma: 28 unidades de saúde; destas, 20 são Unidades de Saúde da Família (USF), 4 são Unidades com Programa de Agentes Comunitários de Saúde (PACS) e 4 são Unidades Básicas de Saúde (UBS). É composto também de 2 unidades de Pronto Atendimento (PA) e 6 centros de referência.

As Unidades de Referência do Programa de Controle da Tuberculose (URPCT) estão localizadas em 2 Unidades de Saúde da Família (USF), 
ou seja, encontram-se inseridas na mesma área física, mas constituem-se URPCT. As equipes destas URPCT são formadas por médico clínico (especialistas em pneumologia ou infectologia), enfermeira, técnico de enfermagem e uma delas possui assistente social. Em 2002 esses serviços notificaram $63 \%$ do total de casos $^{22}$. O Hospital Universitário Cassiano Antônio Moraes (HUCAM referência estadual ambulatorial e hospitalar) contribuiu com $24 \%$ dos casos, e o restante da rede pública hospitalar, com $4 \%$. Somente $10 \%$ dos casos foram notificados pelas UBS do município, que têm o hábito de encaminhar os pacientes para diagnóstico e tratamento para as URPCT.

Utilizou-se a função "esampsi" do Programa Stata versão 10.0 para realizar o cálculo do tamanho amostral e do poder de estudo, e para não se arbitrar nenhum valor empregou-se o valor máximo de prevalência (50\%) da dificuldade de acesso ao diagnóstico da TB com precisão absoluta de $7,0 \%$, um efeito de desenho de 1 , tendo como base a população de 180 pacientes diagnosticados como TB no Município de Vitória, no ano de 2008. O tamanho amostral calculado foi de 88 pacientes e, assumindo-se perdas em torno de $10 \%$, obteve-se o quantitativo final de 97.

Os dados foram coletados através de fontes primárias (entrevistas pré-agendadas com doentes, utilizando instrumento de coleta de dados semiestruturado) e fontes secundárias de informações (prontuários e fichas de notificação), no período de janeiro a dezembro de 2009.

Os critérios de inclusão foram: idade superior a 18 anos, residentes no município de Vitória, diagnosticados entre janeiro a dezembro de 2009, com tempo de tratamento inferior a um mês, que concordaram em participar do estudo conforme o Termo de Consentimento Livre e Esclarecido (TCLE) apresentado.

Os critérios de exclusão foram: pacientes diagnosticados com TB menores de 18 anos, pacientes psiquiátricos, pacientes diagnosticados no sistema prisional, pacientes que faleceram antes da realização da entrevista e aqueles que já haviam terminado o tratamento no período da coleta de dados.

Utilizou-se o instrumento Primary Care Assessment Tool (PCAT), elaborado por Viacava et al. ${ }^{23}$, adaptado e validado para o Brasil por Almeida e $\mathrm{Macinko}^{24}$ e adaptado para a atenção à TB por Villa e Ruffino-Netto ${ }^{25}$. Esse instrumento de coleta de dados contempla questões referentes às características sociodemográficas, caracterizando o perfil do doente de TB, e questões sobre cada dimensão essencial da Atenção Pri- mária à Saúde - APS (porta de entrada, acesso, vínculo, coordenação, enfoque na família, elenco de serviços e formação profissional). Para análise da acessibilidade ao diagnóstico da TB, foram considerados aspectos sociodemográficos e clínicos dos doentes de TB e questões alocadas nas dimensões porta de entrada e acesso.

Para apresentação dos dados, as variáveis cujas respostas eram baseadas em uma escala de Likert (de 1 a 5) foram agrupadas da seguinte forma: variáveis cuja escala consistia em $1=\mathrm{Nun-}$ ca, $2=$ Quase Nunca, $3=$ Às vezes, $4=$ Quase sempre, $5=$ Sempre, o valor 1 corresponde a Não, e os valores 2 a 5 correspondem a Sim; variáveis cuja escala consistia em $1=$ Muito Distante, $2=$ Distante, $3=$ Regular, $4=$ Próximo, $5=$ Muito próxi$m o$, os valores 1 a 2 correspondem a Distante, e os valores 3 a 5 correspondem a Próximo.

Para analisar a acessibilidade aos serviços de saúde para o diagnóstico da TB, estes foram agrupados em quatro estratos: serviços de Atenção Básica (AB) (UBS + USF), PA, URPCT e outros serviços (hospitais, consultórios particulares).

As entrevistas foram realizadas por meio de um agendamento, via telefone ou através dos próprios profissionais do serviço. A aplicação do questionário foi realizada na própria unidade de saúde, sendo a entrevista feita em local que proporcionasse privacidade ao entrevistado.

A análise estatística foi realizada com técnicas de estatística descritiva, com análise de frequências absolutas e relativas, e análise bivariada utilizando o teste qui-quadrado utilizando-se uma significância de $5 \%(\mathrm{p}<0,05)$.

As análises foram desenvolvidas com o programa Statistica 9.0 (Statsoft).

Este projeto foi submetido e aprovado pela Prefeitura Municipal de Vitória e pelo Comitê de Ética da Universidade Federal do Espírito Santo.

\section{Resultados}

No período de janeiro a dezembro de 2009, 142 doentes realizaram tratamento para $\mathrm{TB}$, em Vitória (ES).

Como já assinalado anteriormente foram excluídos do estudo pacientes diagnosticados com TB menores de 18 anos (7), pacientes psiquiátricos (2), pacientes diagnosticados no sistema prisional (8), pacientes que faleceram antes da realização da entrevista e (3) aqueles que já haviam terminado o tratamento no período da coleta de dados (21). O total de pacientes na pesquisa foi de 101. 
A Tabela 1 apresenta os dados sobre perfil sociodemográfico e clínico dos doentes de TB incluídos no estudo.

Tabela 1. Distribuição de frequências das variáveis sociodemográficas e clínicas dos doentes de TB no município de Vitória, ES, 2009.

\begin{tabular}{|c|c|c|}
\hline Variáveis & $\mathbf{n}$ & $\%$ \\
\hline \multicolumn{3}{|l|}{ Sexo } \\
\hline Feminino & 41 & 40,6 \\
\hline Masculino & 60 & 59,4 \\
\hline \multicolumn{3}{|l|}{ Faixa etária } \\
\hline 18 a 29 anos & 31 & 30,69 \\
\hline 30 a 39 anos & 27 & 26,73 \\
\hline 40 a 49 anos & 18 & 17,82 \\
\hline 50 a 59 anos & 18 & 17,82 \\
\hline Acima de 60 anos & 07 & 6,93 \\
\hline \multicolumn{3}{|l|}{ Escolaridade } \\
\hline Sem escolaridade ou EF incompleto & 62 & 61,38 \\
\hline EF completo ou mais & 39 & 38,61 \\
\hline \multicolumn{3}{|l|}{ Situação empregatícia } \\
\hline Com renda & 94 & 93,06 \\
\hline Sem renda & 7 & 6,93 \\
\hline \multicolumn{3}{|l|}{ Renda familiar categorizada } \\
\hline Sem rendimento & 7 & 6,93 \\
\hline Até $2 \mathrm{SM}^{*}$ & 62 & 61,38 \\
\hline De 2 a 5 SM & 16 & 15,84 \\
\hline Maior 5 SM & 16 & 15,84 \\
\hline \multicolumn{3}{|l|}{ Forma clínica da doença } \\
\hline Pulmonar & 86 & 85,14 \\
\hline Extrapulmonar & 11 & 10,89 \\
\hline Pulmonar + extrapulmonar & 4 & 3,96 \\
\hline \multicolumn{3}{|l|}{ Tipo de caso } \\
\hline Casos novos & 88 & 87,12 \\
\hline Recidiva/retratamento & 13 & 12,87 \\
\hline \multicolumn{3}{|l|}{ Coinfecção TB/HIV } \\
\hline Sim & 3 & 2,97 \\
\hline Não & 85 & 84,15 \\
\hline Ignorado & 13 & 12,87 \\
\hline Total & 101 & 100,0 \\
\hline
\end{tabular}

Dos 101 doentes entrevistados, 59,4\% eram do sexo masculino; a faixa etária predominante encontrada foi de 18 a 29 anos com 30,6\%, conforme Tabela 1. Encontrou-se doente que se enquadrava na faixa etária dos 80 anos, sendo essa a maior idade encontrada.

Em relação à escolaridade, a maioria dos doentes se enquadra na categoria desprovida de escolaridade ou com um ensino fundamental incompleto $(61,3 \%)$, sendo que 93,0\% apresentavam situação empregatícia com renda.

A predominância da renda mensal familiar encontrada foi de até $2 \mathrm{SM}$, em que $61,3 \%$ dos doentes se encontravam nessa faixa salarial.

Em relação às características clínicas dos doentes, encontrou-se que $85,1 \%$ apresentava tuberculose pulmonar; $87,1 \%$ eram casos novos e $84,1 \%$ não eram coinfectados com o HIV. Ressalta-se que $12,8 \%$ dos doentes não tinham o resultado do teste anti-HIV, conforme Tabela 1.

O primeiro tipo de Serviço de Saúde (SS) mais procurado pelo doente quando começou a perceber os sinais e sintomas da doença foi a Atenção Básica (AB) com 38/101 (37,6\%), seguida pelo PA com 31/101 (30,7\%). Em contrapartida, o serviço de saúde que mais diagnosticou a TB entre os doentes entrevistados foi a URPCT com $62 / 101(61,3 \%)$, seguido pela AB com 20/101 $(19,8 \%)$, conforme Tabela 2.

Observou-se que apenas 43/101 (42,5\%) dos doentes foram diagnosticados no primeiro tipo de SS procurado. A maioria dos doentes que procuraram o PA como primeira escolha foi diagnosticada na URPCT $(54,8 \%)$. Dos doentes que procuraram a $\mathrm{AB}$, apenas $36,8 \%$ obtiveram o diagnóstico no mesmo local procurado. Todos os doentes que procuraram a URPCT foram diagnosticados no mesmo serviço (Tabela 2).

Os aspectos organizacionais dos doentes de TB são apresentados na Tabela 3.

Tabela 2. Distribuição dos doentes de TB entrevistados segundo o primeiro tipo de Serviço de Saúde procurado e o local diagnóstico da TB em Vitória, ES, 2009.

\begin{tabular}{|c|c|c|c|c|c|}
\hline \multirow[b]{2}{*}{$\begin{array}{c}\text { Local de } \\
\text { diagnóstico }\end{array}$} & \multicolumn{4}{|c|}{ Primeiro tipo de Serviço de Saúde procurado } & \multirow[b]{2}{*}{$\begin{array}{r}\text { Total } \\
\text { N (\%) }\end{array}$} \\
\hline & $\begin{array}{c}\text { AB } \\
\mathrm{N}(\%)\end{array}$ & $\begin{array}{l}\text { URPCT } \\
\text { N (\%) }\end{array}$ & $\begin{array}{c}\text { PA } \\
\text { N (\%) }\end{array}$ & $\begin{array}{l}\text { Outros } \\
\text { N (\%) }\end{array}$ & \\
\hline $\mathrm{AB}$ & $14(36,8)$ & $0(0,0)$ & $5(16,1)$ & $1(7,1)$ & $20(19,8)$ \\
\hline URPCT & $20(52,6)$ & $18(100,0)$ & $17(54,8)$ & $7(50)$ & $62(61,3)$ \\
\hline PA & $3(7,8)$ & $0(0,0)$ & $5(16,1)$ & $0(0,0)$ & $8(7,9)$ \\
\hline Outros & $1(2,6)$ & $0(0,0)$ & $4(12,9)$ & $6(42,8)$ & $11(10,8)$ \\
\hline Total & $38(100,0)$ & $18(100,0)$ & $31(100,0)$ & $14(100,0)$ & $101(100,0)$ \\
\hline
\end{tabular}

Legenda: AB - Atenção Básica, URPCT - Unidade de Referência no Programa de Controle da Tuberculose, PA - ProntoAtendimento. 
Tabela 3. Análise entre o primeiro tipo de Serviço de Saúde procurado pelo doente e os aspectos organizacionais, Vitória, ES, 2009.

\begin{tabular}{|c|c|c|c|c|c|}
\hline \multirow[b]{2}{*}{ Variáveis } & \multicolumn{4}{|c|}{ Primeiro tipo de Serviço de Saúde procurado pelo doente } & \multirow[b]{2}{*}{ p-valor } \\
\hline & $\begin{array}{c}\text { AB } \\
\text { n (\%) }\end{array}$ & $\begin{array}{c}\text { PA } \\
\text { n (\%) }\end{array}$ & $\begin{array}{c}\text { URPCT } \\
\text { n (\%) }\end{array}$ & $\begin{array}{c}\text { Outros } \\
\text { n (\%) }\end{array}$ & \\
\hline \multicolumn{6}{|l|}{ Aspectos Organizacionais } \\
\hline $\begin{array}{l}\text { Tempo de demora na } \\
\text { obtenção de consulta no } \\
\text { primeiro tipo de Serviço de } \\
\text { Saúde procurado (dias) }\end{array}$ & & & & & 0,0182 \\
\hline $0($ dia $)$ & $25(65,7)$ & $31(100,0)$ & $16(88,8)$ & $10(71,4)$ & \\
\hline $1-7$ (dias) & $11(28,9)$ & $0(0,0)$ & $2(11,1)$ & $3(21,4)$ & \\
\hline $8-30($ dias $)$ & $2(5,2)$ & $0(0,0)$ & $0(0,0)$ & $1(7,1)$ & \\
\hline $\begin{array}{l}\text { Hipótese diagnóstica } \\
\text { realizada pelo primeiro tipo } \\
\text { de Serviço de Saúde } \\
\text { procurado }\end{array}$ & & & & & 0,0001 \\
\hline Suspeita de TB & $8(21,0)$ & $10(32,2)$ & $16(88,8)$ & $7(50,0)$ & \\
\hline Suspeita de outra doença & $10(26,3)$ & $12(38,7)$ & $1(5,5)$ & $3(21,4)$ & \\
\hline Não mencionado & $20(52,6)$ & $9(29,0)$ & $1(5,5)$ & $4(28,5)$ & \\
\hline $\begin{array}{l}\text { Solicitação de exame de } \\
\text { escarro }\end{array}$ & & & & & 0,0003 \\
\hline Sim & $16(42,1)$ & $8(25,8)$ & $16(88,8)$ & $6(42,8)$ & \\
\hline Não & $22(57,9)$ & $23(74,2)$ & $2(11,2)$ & $8(57,2)$ & \\
\hline $\begin{array}{l}\text { Solicitação de exame de } \\
\text { Raios-x }\end{array}$ & & & & & 0,0159 \\
\hline Sim & $23(60,5)$ & $23(74,2)$ & $17(94,4)$ & $13(92,8)$ & \\
\hline Não & $15(39,5)$ & $8(25,8)$ & $1(5,6)$ & $1(7,2)$ & \\
\hline $\begin{array}{l}\text { Encaminhamento para outro } \\
\text { profissional }\end{array}$ & & & & & 0,0846 \\
\hline Sim & $16(42,1)$ & $14(45,1)$ & $2(11,1)$ & $6(42,8)$ & \\
\hline Não & $22(57,9)$ & $17(54,9)$ & $16(88,9)$ & $8(57,2)$ & \\
\hline $\begin{array}{l}\text { Encaminhamento para } \\
\text { realização de exame de } \\
\text { escarro }\end{array}$ & & & & & 0,6018 \\
\hline Sim & $5(13,1)$ & $5(16,1)$ & $1(5,5)$ & $3(21,4)$ & \\
\hline Não & $33(86,9)$ & $26(83,9)$ & $17(94,4)$ & $11(78,6)$ & \\
\hline $\begin{array}{l}\text { Encaminhamento para } \\
\text { realização de Raios-X em } \\
\text { outro serviço }\end{array}$ & & & & & 0,0001 \\
\hline Sim & $21(55,2)$ & $1(3,2)$ & $17(94,4)$ & $2(14,2)$ & \\
\hline Não & $17(44,8)$ & $30(96,8)$ & $1(5,5)$ & $12(85,8)$ & \\
\hline $\begin{array}{l}\text { Diagnóstico no primeiro } \\
\text { tipo de Serviço de Saúde } \\
\text { procurado }\end{array}$ & & & & & 0,0001 \\
\hline Sim & $14(36,8)$ & $5(16,1)$ & $18(100,0)$ & $5(35,7)$ & \\
\hline Não & $24(63,20)$ & $26(83,9)$ & $0(0,0)$ & $9(64,3)$ & \\
\hline $\begin{array}{l}\text { Facilidade na obtenção de } \\
\text { consulta no Serviço de Saúde } \\
\text { que descobriu a TB }\end{array}$ & & & & & 0,3695 \\
\hline Sim & $36(94,7)$ & $28(90,3)$ & $18(100,0)$ & $14(100,0)$ & \\
\hline Não & & $3(9,7)$ & $0(0,0)$ & $0(0,0)$ & \\
\hline
\end{tabular}


Tabela 3. continuação

\begin{tabular}{|c|c|c|c|c|c|}
\hline \multirow[b]{2}{*}{ Variáveis } & \multicolumn{4}{|c|}{ Primeiro tipo de Serviço de Saúde procurado pelo doente } & \multirow[b]{2}{*}{ p-valor } \\
\hline & $\begin{array}{c}\mathrm{AB} \\
\mathbf{n}(\%)\end{array}$ & $\begin{array}{c}\text { PA } \\
\text { n (\%) }\end{array}$ & $\begin{array}{c}\text { URPCT } \\
\text { n (\%) }\end{array}$ & $\begin{array}{c}\text { Outros } \\
\text { n (\%) }\end{array}$ & \\
\hline $\begin{array}{l}\text { Horários de atendimento, } \\
\text { nos finais de semana, do } \\
\text { Serviço de Saúde que } \\
\text { descobriu a TB }\end{array}$ & & & & & 0,0807 \\
\hline Sim & $5(13,1)$ & $9(29,0)$ & $1(5,5)$ & $6(42,8)$ & \\
\hline Não & $19(50,0)$ & $10(32,2)$ & $7(38,8)$ & $5(35,7)$ & \\
\hline Não sabe & $14(36,8)$ & $12(38,7)$ & $10(55,5)$ & $3(21,4)$ & \\
\hline $\begin{array}{l}\text { Horários de atendimento do } \\
\text { Serviço de Saúde que } \\
\text { descobriu a TB, depois das } \\
18 \text { h, pelo menos } 1 \text { dia } \\
\text { durante a semana }\end{array}$ & & & & & 0,2495 \\
\hline Sim & $10(26,3)$ & $11(35,4)$ & $3(16,6)$ & $6(42,8)$ & \\
\hline Não & $10(26,3)$ & $11(35,4)$ & $3(16,6)$ & $4(28,5)$ & \\
\hline Não sabe & $18(47,3)$ & $17(54,8)$ & $12(66,6)$ & $4(28,5)$ & \\
\hline $\begin{array}{l}\text { Exames realizados no } \\
\text { próprio Serviço de Saúde que } \\
\text { diagnosticou a TB }\end{array}$ & & & & & 0,0018 \\
\hline Sim & $3(7,8)$ & $8(25,8)$ & $1(5,5)$ & $7(50,0)$ & \\
\hline Não & $35(92,20)$ & $23(74,2)$ & $17(94,5)$ & $7(50,0)$ & \\
\hline $\begin{array}{l}\text { Recebimento de orientações } \\
\text { para realização dos exames }\end{array}$ & 3 & & & & 0,3853 \\
\hline $\begin{array}{l}\text { Sim } \\
\text { Não }\end{array}$ & $\begin{array}{r}6(94,7) \\
2(5,3)\end{array}$ & $\begin{array}{r}28(90,3) \\
3(9,7)\end{array}$ & $\begin{array}{r}18(100,0) \\
0(0,0)\end{array}$ & $\begin{array}{r}12(85,7) \\
2(14,3)\end{array}$ & \\
\hline $\begin{array}{l}\text { Frequência com que faltaram } \\
\text { materiais para a realização } \\
\text { dos exames no Serviço de } \\
\text { Saúde que diagnosticou a TB }\end{array}$ & & & & & 0,7724 \\
\hline Sim & $1(2,6)$ & $1(3,2)$ & $0(0,0)$ & $0(0,0)$ & \\
\hline Não & $37(97,3)$ & $29(93,5)$ & $18(100,0)$ & $14(100,0)$ & \\
\hline Não sabe & $0(0,0)$ & $1(3,2)$ & $0(0,0)$ & $0(0,0)$ & \\
\hline $\begin{array}{l}\text { Dificuldade encontrada para } \\
\text { a entrega do pote de escarro }\end{array}$ & & & & & 0,3313 \\
\hline $\operatorname{Sim}$ & $4(10,5)$ & $5(16,1)$ & $0(0,0)$ & $1(7,1)$ & \\
\hline Não & $34(89,5)$ & $26(83,9)$ & $18(100,0)$ & $13(92,9)$ & \\
\hline $\begin{array}{l}\text { Serviço de Saúde que } \\
\text { informou sobre os resultados } \\
\text { dos exames foi o mesmo que } \\
\text { os solicitou }\end{array}$ & & & & & 0,0616 \\
\hline Sim & $35(92,1)$ & $25(80,6)$ & $18(100,0)$ & $14(100,0)$ & \\
\hline Não & $3(7,9)$ & $6(19,4)$ & $0(0,0)$ & $0(0,0)$ & \\
\hline
\end{tabular}

Dos doentes que procuraram a URPCT, 16/ $18(88,8 \%)$ obtiveram consulta imediatamente, enquanto que $11 / 38(28,9 \%)$ daqueles que procuraram os serviços da $\mathrm{AB}$ obtiveram a consulta entre 1 a 7 dias, sendo essa diferença estatisticamente significativa $(p=0,0182)$ a associação entre o primeiro tipo de SS procurado e o tempo de demora na obtenção de consulta nesse serviço, conforme Tabela 3.

Quanto à hipótese diagnóstica realizada pelo primeiro tipo de SS procurado, dos doentes que procuraram a URPCT, 16/18 (88,8\%) foram considerados com suspeição de TB, enquanto que apenas 10/31 (32,2\%) daqueles que procuraram 
Tabela 3. continuação

\begin{tabular}{|c|c|c|c|c|c|}
\hline \multirow[b]{2}{*}{ Variáveis } & \multicolumn{4}{|c|}{ Primeiro tipo de Serviço de Saúde procurado pelo doente } & \multirow[b]{2}{*}{ p-valor } \\
\hline & $\begin{array}{c}\text { AB } \\
\mathbf{n}(\%)\end{array}$ & $\begin{array}{c}\text { PA } \\
\mathbf{n}(\%)\end{array}$ & $\begin{array}{l}\text { URPCT } \\
\text { n (\%) }\end{array}$ & $\begin{array}{l}\text { Outros } \\
\text { n (\%) }\end{array}$ & \\
\hline $\begin{array}{l}\text { Exames realizados para o } \\
\text { diagnóstico da TB }\end{array}$ & & & & & 0,3083 \\
\hline \multicolumn{6}{|l|}{ Baciloscopia } \\
\hline Sim & $36(94,7)$ & $28(90,3)$ & $17(94,4)$ & $11(78,5)$ & \\
\hline Não & $2(5,3)$ & $3(9,7)$ & $1(5,6)$ & $3(21,5)$ & \\
\hline PPD & & & & & 0,7584 \\
\hline Sim & $16(42,1)$ & $11(35,4)$ & $8(44,4)$ & $4(28,5)$ & \\
\hline Não & $22(57,9)$ & $20(64,6)$ & $10(55,6)$ & $10(71,5)$ & \\
\hline Raio-X & & & & & 0,4964 \\
\hline Sim & $36(94,7)$ & $30(96,7)$ & $16(88,8)$ & $14(100,0)$ & \\
\hline Não & $2(5,3)$ & $1(3,3)$ & $2(11,2)$ & $0(0,0)$ & \\
\hline Biópsia & & & & & 0,0381 \\
\hline Sim & $1(2,6)$ & $4(12,9)$ & $1(5,5)$ & $4(28,5)$ & \\
\hline Não & $37(97,4)$ & $27(87,1)$ & $17(94,5)$ & $10(71,5)$ & \\
\hline Cultura de escarro & & & & & 0,0529 \\
\hline Sim & $35(92,1)$ & $28(90,3)$ & $16(88,8)$ & $9(64,2)$ & \\
\hline Não & $3(7,9)$ & $3(9,7)$ & $2(11,2)$ & $5(35,8)$ & \\
\hline Anti-HIV & & & & & 0,4737 \\
\hline Sim & $37(97,3)$ & $29(93,5)$ & $17(94,5)$ & $12(85,7)$ & \\
\hline Não & $1(2,6)$ & $2(6,5)$ & $1(5,5)$ & $2(14,3)$ & \\
\hline
\end{tabular}

Legenda: AB - Atenção Básica, URPCT - Unidade de Referência no Programa de Controle da Tuberculose, PA Pronto-Atendimento.

os serviços do PA foram diagnosticados como suspeitos de TB, sendo estatisticamente significativa $(\mathrm{p}=0,0001)$.

Observou-se uma baixa solicitação de baciloscopia pelos serviços de AB 16/38 (42,1\%) e, principalmente, do PA com apenas $8 / 31$ (25,8\%), enquanto que os serviços da URPCT solicitaram 16/18 (88,8\%) de baciloscopia, sendo estatisticamente significativa $(\mathrm{p}=0,0003)$.

Dos doentes que tiveram o exame de escarro solicitado em qualquer um dos quatro tipos de SS 46/101 (45,5\%), 8/46 (17,3\%) foram encaminhados a outro serviço para a realização deste. Já os doentes que tiveram a URPCT como o primeiro tipo de SS procurado 18/101 (17,8\%), apenas $2 / 18(11,1 \%)$ tiveram que ser encaminhados a outros serviços para consulta médica com outro profissional.

A URPCT foi o tipo de SS que mais solicitou exame de Raio-X 17/18 (94,4\%) neste serviço, enquanto que a $\mathrm{AB}$ solicitou $23 / 38(60,5 \%)$, sendo estatisticamente significativo $(\mathrm{p}=0,0159)$

A URPCT também foi o tipo de SS que mais encaminhou para a realização de Raio-X em outro serviço entre aqueles usuários que procuraram pelo primeiro atendimento $17 / 18(94,4 \%)$ neste serviço. Já a $\mathrm{AB}$ encaminhou 21/ 38 (55,2\%), sendo estatisticamente significativo $(p=0,0001)$.

O PA foi um dos SS que menos diagnosticou aqueles usuários que procuraram pelo primeiro atendimento $5 / 31(16,1 \%)$ neste serviço, enquanto que $18 / 18$ (100\%) daqueles que procuraram os serviços da URPCT obtiveram o diagnóstico, sendo essa diferença estatisticamente significativa $(\mathrm{p}=0,0001)$, conforme a Tabela 3 .

Quanto aos exames realizados no próprio SS que diagnosticou a TB, dos doentes que procuraram a URPCT apenas $1 / 18(5,5 \%)$ realizou os exames no mesmo serviço, seguidos pelos $3 / 38$ $(7,8 \%)$ daqueles que procuraram os serviços da $\mathrm{AB}$, enquanto que aqueles que procuraram outros serviços $7 / 14(50 \%)$ realizaram os exames no mesmo SS procurado (Tabela 3 ).

Em relação ao recebimento de orientações para a realização dos exames, a maioria dos doentes que procuraram a $\mathrm{AB}$, o PA e outros serviços, afirmaram ter recebido instruções, sendo que todos os doentes que procuraram a URPCT como primeiro tipo de SS 18/18 (100,0\%) receberam orientações para a realização dos exames, sendo sem significância estatística $(\mathrm{p}=0,3853)$.

Observou-se, ainda, que raramente faltaram materiais para a realização dos exames nos serviços $(\mathrm{p}=0,7724)$ e que a maioria dos doentes 
foi informada sobre os resultados dos exames realizados nos respectivos serviços que os solicitaram $(\mathrm{p}=0,0616)$.

As variáveis pertinentes aos aspectos geográficos dos doentes de TB são apresentadas na Tabela 4 .

Dos doentes que procuraram a $\mathrm{AB}, 35 / 38$ $(92,1 \%)$ relataram morar próximo das unidades, enquanto que $15 / 18(83,3 \%)$ daqueles que procuraram os serviços da URPCT consideravam seus domicílios distantes do local procurado (Tabela 4).

Dos doentes que procuraram a $\mathrm{AB}, 27 / 38$ $(71,0 \%)$ utilizavam transporte motorizado no deslocamento até o SS para o diagnóstico da TB, enquanto que aqueles que procuraram os serviços da URPCT apenas 4/18 (22,2\%) utilizavam transporte motorizado (Tabela 4).

Os aspectos econômicos dos doentes de TB são apresentados na Tabela 5 .

Quanto à perda de turno de trabalho para ir ao SS, observou-se que apenas $56,4 \%$ não foram prejudicados em seu turno de trabalho ou compromisso. Além disso, a maioria dos doentes gastou dinheiro com transporte para ir ao SS, correspondendo a $63,3 \%$.

Observou-se, ainda, que $99,0 \%$ não precisaram de pagar para ser atendidos no SS que diagnosticou a TB e que, apenas $7,9 \%$ dos doentes necessitaram de ajuda financeira para realizar consultas/exames.

\section{Discussão}

Os resultados evidenciam que o primeiro tipo de SS mais procurado pelo doente foi a $A B$, o que pode ser compreendido devido à USF ter alta cobertura em Vitória e ter boa resolutividade, inclusive, para encaminhamentos de referência e contrarreferência. De acordo com o Índice de Desempenho do Sistema Único de Saúde (IDSUS), Vitória apresenta o melhor acesso ao serviço de saúde do país ${ }^{26}$.

Entretanto, no Município de Vitória (ES), a descentralização das ações de controle da TB vem se moldando de forma lenta à medida que se reorganiza a rede de assistência à saúde, articula-

Tabela 4. Análise entre o primeiro tipo de Serviço de Saúde procurado pelo doente e os aspectos geográficos, Vitória, ES, 2009.

\begin{tabular}{|c|c|c|c|c|c|}
\hline \multirow[b]{2}{*}{ Variáveis } & \multicolumn{4}{|c|}{ Primeiro tipo de Serviço de Saúde procurado pelo doente } & \multirow[b]{2}{*}{ p-valor } \\
\hline & $\begin{array}{c}\mathrm{AB} \\
\mathbf{n}(\%)\end{array}$ & $\begin{array}{c}\text { PA } \\
\text { n (\%) }\end{array}$ & $\begin{array}{c}\text { URPCT } \\
\text { n (\%) }\end{array}$ & $\begin{array}{c}\text { Outros } \\
\text { n (\%) }\end{array}$ & \\
\hline \multicolumn{6}{|l|}{ Aspectos geográficos } \\
\hline Proximidade do domicílio & & & & & 0,0001 \\
\hline Sim & $35(92,1)$ & $8(25,8)$ & $3(16,6)$ & $5(35,7)$ & \\
\hline Não & $3(7,8)$ & $23(74,1)$ & $15(83,3)$ & $9(64,2)$ & \\
\hline $\begin{array}{l}\text { Distância do domicílio do } \\
\text { doente até o serviço de saúde } \\
\text { que diagnosticou a TB }\end{array}$ & & & & & 0,0577 \\
\hline Distante & $5(13,1)$ & $12(38,7)$ & $5(27,7)$ & $6(42,8)$ & \\
\hline Próximo & $33(86,8)$ & $19(61,2)$ & $13(72,2)$ & $8(57,1)$ & \\
\hline $\begin{array}{l}\text { Meio de transporte mais } \\
\text { utilizado para ir até o serviço } \\
\text { de saúde para descobrir a TB }\end{array}$ & & & & & 0,0658 \\
\hline Motorizado & $20(52,6)$ & $25(80,6)$ & $11(61,1)$ & $11(78,5)$ & \\
\hline Não motorizado & $18(47,3)$ & $6(19,3)$ & $7(38,8)$ & $3(21,4)$ & \\
\hline $\begin{array}{l}\text { Utilização de transporte } \\
\text { motorizado para ir até o } \\
\text { serviço de saúde para } \\
\text { descobrir a TB }\end{array}$ & & & & & 0,3782 \\
\hline Sim & $27(71,0)$ & $27(87,1)$ & $14(77,7)$ & $12(85,7)$ & \\
\hline Não & $11(28,9)$ & $4(12,9)$ & $4(22,2)$ & $2(14,2)$ & \\
\hline
\end{tabular}

Legendas: AB - Atenção Básica, URPCT - Unidade de Referência no Programa de Controle da Tuberculose, PA

- Pronto-Atendimento. 
Tabela 5. Análise entre o primeiro tipo de Serviço de Saúde procurado pelo doente e os aspectos econômicos, Vitória, ES, 2009.

\begin{tabular}{|c|c|c|c|c|c|}
\hline \multirow[b]{2}{*}{ Variáveis } & \multicolumn{4}{|c|}{ Primeiro tipo de Serviço de Saúde procurado pelo doente } & \multirow[b]{2}{*}{ p-valor } \\
\hline & $\begin{array}{c}\mathrm{AB} \\
\mathbf{n}(\%)\end{array}$ & $\begin{array}{c}\text { PA } \\
\text { n }(\%)\end{array}$ & $\begin{array}{l}\text { URPCT } \\
\text { n (\%) }\end{array}$ & $\begin{array}{l}\text { Outros } \\
\text { n }(\%)\end{array}$ & \\
\hline \multicolumn{6}{|l|}{ Aspectos econômicos } \\
\hline $\begin{array}{l}\text { Perda de turno de trabalho } \\
\text { para ir ao serviço de saúde }\end{array}$ & & & & & 0,2437 \\
\hline $\operatorname{Sim}$ & $16(42,1)$ & $15(48,3)$ & $10(55,5)$ & $3(21,4)$ & \\
\hline Não & $22(57,8)$ & $16(51,6)$ & $8(44,4)$ & $11(78,5)$ & \\
\hline $\begin{array}{l}\text { Gasto de dinheiro com } \\
\text { transporte para ir ao SS }\end{array}$ & & & & & 0,8650 \\
\hline Sim & $24(63,1)$ & $21(67,7)$ & $10(55,5)$ & $9(64,2)$ & \\
\hline Não & $14(36,8)$ & $10(32,2)$ & $8(44,4)$ & $5(35,7)$ & \\
\hline \multicolumn{6}{|l|}{ Pagamento para ser atendido } \\
\hline no SS que diagnosticou a TB & & & & & 0,0989 \\
\hline Sim & $0(0,0)$ & $0(0,0)$ & $0(0,0)$ & $1(7,1)$ & \\
\hline Não & $38(100,0)$ & $31(100,0)$ & $18(100,0)$ & $13(92,8)$ & \\
\hline \multicolumn{6}{|l|}{ Necessidade de ajuda } \\
\hline $\begin{array}{l}\text { financeira para realizar } \\
\text { consultas/exames }\end{array}$ & & & & & 0,2054 \\
\hline Sim & $3(7,8)$ & $1(3,2)$ & $1(5,5)$ & $3(21,4)$ & \\
\hline Não & $35(92,1)$ & $30(96,7)$ & $17(94,4)$ & $11(78,5)$ & \\
\hline
\end{tabular}

Legendas: AB - Atenção Básica, URPCT - Unidade de Referência no Programa de Controle da Tuberculose, PA

- Pronto-Atendimento.

da, sobretudo, com a ampliação da cobertura do PSF/PACS. Apesar das URPCT funcionarem fisicamente em Unidades de Saúde da Família (USF), a organização do serviço é de nível secundário de atenção.

É importante ressaltar que, apesar disso, uma parte considerável dos doentes $(30,7 \%)$, neste estudo, procurou como primeiro atendimento o PA. Esta escolha pode ter sido motivada pelo fato dos usuários do SUS procurarem mais os serviços de saúde por motivo de doença e optarem pela utilização mais frequente de hospitais e prontos-socorros ${ }^{27}$. Outra hipótese poderia ser explicada pelo fato de que os pacientes encontram maior flexibilidade de horários para serem atendidos nesse serviço, uma vez que a preferência pelo PA decorre do horário restrito de funcionamento dos serviços de atenção primária ${ }^{28}$.

Embora a $\mathrm{AB}$ tenha sido o primeiro tipo de SS mais procurado pelos doentes, o diagnóstico da TB predominou nas URPCT, seguido pelos serviços de $A B$, sendo que todos os doentes que procuraram a URPCT foram diagnosticados no mesmo serviço, enquanto que poucos doentes foram diagnosticados em serviços privados. A partir desse ponto, é importante destacar que os doentes, muitas vezes, procuram por atendimento nas Unidades Básicas de Saúde e, devido ao fato de não encontrarem resolutividade para a sua sintomatologia nesse nível de atenção, são encaminhados aos serviços secundários para a realização do diagnóstico da doença. Em um estudo realizado por Oliveira et al. ${ }^{28}$, evidenciou-se que a maior parte dos diagnósticos de TB foi realizada em níveis secundários e terciários de atenção (Pronto-Atendimento).

Outro fator que pode ter influenciado na acessibilidade são os horários rígidos e limitados e as formas quase sempre presenciais de marcação de consultas que podem dificultar o efetivo acesso à atenção básica, especialmente nas metrópoles, em que parcelas importantes da população passam o dia longe de seu local de moradia ${ }^{23}$. Além disso, reconhece-se a necessidade de proporcionar aos profissionais de saúde uma capacitação mais aprofundada sobre o diagnóstico da TB a fim de que os casos sejam diagnosticados precocemente não só nas URPCT, mas em todos os serviços de saúde.

Recentemente, um estudo realizado em Vitória (ES) mostrou que dos 164 entrevistados que relataram tosse, $35(21,3 \%)$ o fizeram esponta- 
neamente, como motivo que os levou a procurar a unidade de saúde, e 129 (78,7\%) só relataram a tosse quando perguntados diretamente. Tal situação foi semelhante em todas as regiões do município ${ }^{29}$.

Um dos pontos que podem ser levantados é que, uma vez que o paciente chega ao serviço ele possui o atendimento necessário à sua demanda, talvez uma das principais dificuldades seja ainda a identificação desses pacientes de forma precoce na comunidade e seu acesso aos serviços de maneira adequada ${ }^{30}$.

A ampliação da realização de baciloscopia para a rede de serviços de saúde é uma das formas para detectar os doentes de tuberculose e iniciar precocemente o tratamento dos $\operatorname{casos}^{31}$. Uma vez que se propõe a expansão deste procedimento, a baixa solicitação de baciloscopia observada pelos serviços de $\mathrm{AB}$ e, principalmente, do PA, sugere que este pode ser alusivo de deficiências organizacionais (previsão de insumos, capacitação insuficiente dos profissionais para realização do procedi-mento) que repercutem no acesso dos doentes ao diagnóstico da TB.

$\mathrm{O}$ estudo mostra que uma parte expressiva dos doentes que tiveram o exame de escarro solicitado em qualquer um dos quatro tipos de SS, foram encaminhados a outro serviço para a realização deste. No entanto, é importante ressaltar que a insuficiente oferta do procedimento de coleta de escarro pelos SS exige deslocamento dos casos suspeitos de TB para outros serviços. Ao transferir para os pacientes a responsabilidade de proceder à coleta e encarregá-los de levar o material ao local estabelecido, coloca-se em risco a ação planejada. Logo, evidencia-se que, apesar da agilidade em se conseguir uma consulta médica em alguns serviços, o diagnóstico e o controle dos casos de TB podem estar sendo prejudicados frente às dificuldades de acesso ao diagnósti$\mathrm{Co}^{31}$, além do itinerário que o usuário precisa de percorrer para obter o seu diagnóstico.

A realização do exame radiológico é outra forma de diagnóstico, indicada para suspeita clínica de TB em pacientes imunodeprimidos e em doentes com sintomas respiratórios e baciloscopia negativa ${ }^{32}$.

Percebe-se que a solicitação do exame de escarro ocorreu apenas para $42,1 \%$ dos doentes que buscaram a $\mathrm{AB}$ como primeiro tipo de SS, enquanto a radiografia ocorreu apenas para $60,5 \%$ desses doentes. Esse resultado pode estar associado à baixa suspeita da TB pelos profissionais de saúde. O alto número de encaminhamentos para realização de Raios-x pode estar relacionado ao tipo de SS procurado, em que a maioria dos estabelecimentos de saúde é desprovida de equipamentos para a realização do exame radiológico.

Assim, nota-se no presente estudo que o uso da radiografia é maior que o do exame de escarro, sendo que este deve ser pedido, mesmo com a solicitação do Raio-x, para identificação dos casos bacilíferos. Acredita-se que quando se trata do atendimento no primeiro tipo de SS procurado pelos doentes, os profissionais de saúde não estejam vigilantes para a TB, assim solicitando inicialmente apenas a radiografia, ampliando as possibilidades diagnósticas, devido à suspeita conjunta de pneumopatias ${ }^{33}$.

Acresce-se que a quantidade reduzida de doentes que tiveram todos os exames realizados no próprio SS que diagnosticou a TB é devido à falta, nesses serviços, de aparelhamentos que possibilitam a execução de todos os exames solicitados.

Um aspecto importante que influencia a qualidade das amostras de escarro é a orientação efetuada pelos profissionais de saúde e sua compreensão pelo paciente ${ }^{29}$. Neste estudo, a maioria dos doentes afirmou ter recebido orientações dos profissionais de saúde para a realização dos exames e foi informada sobre os respectivos resultados nos serviços que os solicitaram, sendo que raramente faltaram materiais para sua concretização.

Apesar daqueles que procuraram a $\mathrm{AB}$ como primeiro serviço de saúde residir próximo a este serviço, isso não implicou diretamente no acesso, pois a $\mathrm{AB}$ foi um dos serviços que mais demorou a realizar o diagnóstico da TB. É necessário considerar, portanto, que apenas a distância do serviço de saúde pode não significar barreira proeminente, uma vez que é observada a preferência de alguns indivíduos pela busca do diagnóstico longe do domicílio, por se sentirem mais confortáveis, devido ao estigma relacionado à doença ${ }^{34}$ ou por acreditarem que sua demanda será mais facilmente atendida. Observa-se, portanto, que a existência de alta acessibilidade geográfica necessariamente não garante a possibilidade de acesso mais rápido ao diagnóstico da TB por parte da população, conforme demonstrado em outros estudos ${ }^{35,36}$.

Os dados do estudo apontam para a perda de turno de trabalho para comparecer às consultas médicas na busca por atendimento para o diagnóstico da TB. A demora no atendimento e os horários incompatíveis com os turnos de trabalho podem dificultar a procura pelo serviço de 
saúde para o diagnóstico, uma vez que os pacientes possuem receio de perder o emprego diante da necessidade de faltas e atrasos ${ }^{37}$. Alguns autores ressaltam que a rigidez na rotina de atendimento, por parte dos serviços de saúde, agrava as dificuldades econômicas e sociais vivenciadas pelos indivíduos portadores de $\mathrm{TB}^{37,38}$.

A identificação de lacunas importantes na acessibilidade ao diagnóstico de TB parece estar rela- cionada às dificuldades operacionais de organização da atenção à saúde. Nesse sentido, mudanças na oferta de radiografia em serviços próximos ao paciente, horários compatíveis com o turno de trabalho, profissionais treinados para identificação precoce dos casos de TB e disponibilidade de recursos materiais para o diagnóstico poderão melhorar o acesso ao diagnóstico de TB e, assim, contribuir com o controle desta doença.

\section{Colaboradores}

RB Loureiro participou da concepção, delineamento, análise e interpretação dos dados, redação do artigo, revisão crítica e aprovação da versão a ser publicada; TCS Villa e A Ruffino-Netto participaram da concepção, delineamento, revisão crítica e aprovação da versão a ser publicada; RL Peres participou da revisão crítica e da aprovação da versão a ser publicada; JU Braga participou da revisão crítica; E Zandonade participou da análise e interpretação dos dados, e da revisão crítica; ELN Maciel participou da concepção, delineamento, análise e interpretação dos dados, revisão crítica e aprovação da versão a ser publicada.

\section{Agradecimentos}

Os autores agradecem o auxílio financeiro recebido pelo International Clinical Operational and Health Services Research and Training Award (ICHORTA-AIDS/TB), pela Fundação de Amparo à Pesquisa de São Paulo (FAPESP) e pelo Conselho Nacional de Desenvolvimento Científico e Tecnológico (CNPq).

\section{Referências}

1. Figueiredo TMRM, Villa TCS, Scatena LM, Gonzáles RIC, Ruffino-Netto A, Nogueira JA, Oliveira AR, Almeida SA. Desempenho da atenção básica no controle da tuberculose. Rev Saude Publica 2009; 43(5):825-831.

2. Fishbein M, Ajzen I. Belief, attitude, intention and behavior: an introduction to theory and research. Massachussets: Addison-Wesley; 1975.

3. Travassos C. Uma revisão sobre os conceitos de acesso e utilização de serviços de saúde. Cad Saude Publica 2004; 20(Supl. 2):S190-S198.

4. Cerqueira MB, Pupo LR. Condições e modos de vida em duas favelas da baixada santista e suas interfaces com o acesso aos serviços de saúde. Rev. Baiana de Saúde Pública 2009; 33(2).

5. Travassos CMR, Castro MSM. Determinantes e desigualdades sociais no acesso e na utilização de serviços de saúde. In: Giovanella L, Escorel S, Lobato LVC, Noronha JC, Carvalho AI, organizadores. Políticas e sistemas de saúde no Brasil. Rio de Janeiro: Editora Fiocruz; 2008. p. 215-243.

6. Abreu-de-Jesus WL, Assis MMA. Revisão sistemática sobre o conceito de acesso nos serviços de saúde: contribuições do planejamento. Cien Saude Colet 2010; 15(1):161-170.

7. Donabedian A. Aspects of medical care administration. Boston: Harvard University Press; 1973.

8. Frenk J. Conceptand measurement of accessibility. Salud Publ. Mex 1985; 27(5):438-453.

9. Palermo PU. A desigualdade social no acesso à saúde na região sul do Brasil [dissertação]. Porto Alegre: Universidade Federal do Rio Grande do Sul; 2005.

10. Fekete MC. Estudo da acessibilidade na avaliação dos serviços de saúde. Brasília: OPAS, OMS; 1992. (Elaboração para o curso sobre Gerência em Unidades Básicas de Saúde do Distrito Sanitário).

11. Starfield B. Atenção primária: equilíbrio entre necessidades de saúde, serviços e tecnologia. Brasília: Unesco; 2002.

12. Travassos C, Martins M. Uma revisão sobre os conceitos de acesso e utilização de serviços de saúde. Cad Saude Publica 2004; 20(Supl. 2):S190-S198. 
13. Unglert CVS. O enfoque da acessibilidade no planejamento da localização e dimensão de serviços de saúde. Rev Saude Publica 1990; 24(6):445-452.

14. Fekete MC. Estudo da acessibilidade na avaliação dos serviços de saúde. Brasília:OPAS; 1997. [Projeto Gerus. Texto de apoio da unidade I]

15. Wang W, Jiang Q, Abdullah ASM, Xu B. Barriers in accessing to tuberculosis care among non-residents in Shangai: a descriptive study of delays in diagnosis. Eur J Publ Health 2007; 17(5):419-423.

16. Díez M, Bleda MJ, Alcaide J, Castells C, Cardenal JI, Dominguez A, Gayoso P, Guitiérrez G, Huerta C, López MJ, Moreno T, Muñoz F, García-Fulgueiras A, Picó M, Pozo F, Quirós JR, Robles F, Sánchez JM, Vanaclocha H, Vega, T. Determinants of health system delay among confirmed tuberculosis cases in Spain. Eur J Publ Health 2005; 15(4):343-349.

17. Yimer S, Bjune G, Alene G. Diagnostic and treatment delay among pulmonary tuberculosis patients in Ethiopia: a cross sectional study. BMC Infectious Diseases 2005; 5:112

18. Leung ECC, Leung CC, Tam CM. Delayed presentation and treatment of newly diagnosed pulmonary tuberculosis patients in Hong Kong. Hong Kong Medical Journal 2007; 13(13):221-227.

19. Santos MAPS, Albuquerque MFPM, Ximenes RAA, Lucana-Silva NLCL, Braga C, Campelo ARL, Dantas OMS, Montarroyos U, Souza WV, Kawasaki AM, Rodrigues LC. Risk factors for treatment delay in pulmonary tuberculosis in Recife, Brazil. BMC Publ Health 2005; 5:25.

20. Wang W, Jiang Q, Abdullah ASM, Xu B. Barriers in accessing to tuberculosis care among non-residents in Shangai: a descriptive study of delays in diagnosis. Eur J Publ Hlth 2007; 17(5):419-423.

21. Instituto Brasileiro de Geografia e Estatística (IBGE). Estimativas das populações residentes, em $1^{\circ}$ de julho de 2009, segundo os municípios. Rio de Janeiro: IBGE; 2009. [Internet]. [acessado 2014 mar 7]. Disponível em: http://www.ibge.gov.br/home/estatistica/populacao/estimativa2009/POP_2009_TCU.pdf

22. Vieira E.M. Avaliação de serviços de saúde. In: Franco LJ, Passos ADC, organizadores. Fundamentos da Epidemiologia. São Paulo: Manole; 2005. p. 337.

23. Viacava F, Almeida C, Caetano R, Fausto M, Macinko J, Martins M, Noronha JC, Novaes HMD, Oliveira ES, Porto SM, Silva LMV, Szwarcwald CL. Uma Metodologia de Avaliação do Desempenho do Sistema de Saúde Brasileiro. Cien Saude Colet 2004; 9(3):711-724.

24. Almeida CM, Macinko JA. Validação de uma Metodologia de avaliação rápida das características organizacionais e do desempenho dos serviços de atenção básica do Sistema Único de Saúde (SUS) em nível local. Brasília: Organização Pan-Americana da Saúde (OPAS); 2006. (Série técnica desenvolvimento de sistemas e serviços de saúde).

25. Villa TCS, Ruffino Netto A. Questionário para avaliação de desempenho de serviços de atenção básica no controle da TB no Brasil. J Bras Pneumol 2009; 35(6):610-612.

26. Brasil. Ministério da Saúde (MS). ISAGS. [Internet]. [acessado 2012 mar 20]. Disponível em: http:/ /www.isags-unasursalud.org/noticias_interna.asp? lang $=1$ \&idArea $=2 \&$ idPai $=2069$
27. Ribeiro MCSA, Barata RB, Almeida MF, Silva ZP. Perfil sociodemográfico e padrão de utilização de serviços de saúde para usuários e não-usuários do SUS - PNAD 2003. Cien Saude Colet 2006; 11(4):10111022.

28. Oliveira MF, Arcêncio RAA, Ruffino-Netto A, Scatena LM, Palha PF, Villa TCS. A porta de entrada para o diagnóstico da tuberculose no Sistema de Saúde de Ribeirão Preto/SP. Rev Esc Enferm USP 2011; 45(4):898-904

29. Moreira CMM, Zandonade E, Lacerda T, Maciel, ELN. Sintomáticos respiratórios nas unidades de atenção primária no Município de Vitória, Espírito Santo, Brasil. Cad Saude Publica 2010; 26(8):16191626.

30. Maciel ELN, Vieira RCA, Milani EC, Brasil M, Fregona G, Dietze R. O agente comunitário de saúde no controle da tuberculose: conhecimentos e percepções. Cad Saude Publica 2008; 24(6):1377-1386.

31. Marcolino ABL, Nogueira JA, Ruffino-Netto A, Moraes RM, Sá LD, Villa TCS, Rolim FJ. Avaliação do acesso às ações de controle da tuberculose no contexto das equipes de saúde da família de Bayeux - PB. Rev Bras Epidemiol 2009; 12(2):144-157.

32. Oliveira MF. Acesso ao diagnóstico da tuberculose em serviços de saúde do município de Ribeirão Preto - SP (2006-2007) [tese]. Ribeirão Preto: Universidade de São Paulo; 2009.

33. Harter J, Nunes BP, Cardozo-Gonzales RI. A utilização da baciloscopia e da radiografia no diagnóstico da tuberculose em pelotas/RS, 2009. Pelotas: XIX CIC: XII ENPOS - II Mostra Científica; 2010.

34. Paixão LMM, Gontijo ED. Perfil de casos de tuberculose notificados e fatores associados ao abandono, Belo Horizonte, MG. Rev Saude Publica 2007; 41(2):205-213

35. Carr-Hill RA, Hardman G, Martin S, Peacock S, Sheldon TA, Smith P. A formula for distributing NHS revenues based on small area use of hospital beds. York: Centre for Health Economics, University of York; 1994.

36. Goddard M, Smith P. Equity of access to health care services: theory and evidence from the UK. Soc Sci Med 2001; 53(9):1149-1162.

37. Dimitrova B, Balabanova D, Atun RA, Levicheva V, Coker RJ. Health service providers perceptions of barriers to tuberculosis care in Russia. Health Policy Plan 2006; 21(4):265-274.

38. Jaiswal A, Singh V, Ogden J, Porter JD, Sharma PP, Sarin R, Arora VK, Jain RC. Adherence to tuberculosis treatment: lessons from the urban setting of Delhi, India. Trop Med Int Health 2003; 8(7):625633.

Artigo apresentado em 18/03/2013

Aprovado em 02/06/2013

Versão final apresentada em 19/06/2013 